\title{
Visualization of the Current-Density Distribution for MCG With WPW Syndrome Patients Using Independent Component Analysis
}

\author{
K. Kobayashi, Y. Uchikawa, Member, IEEE, K. Nakai, and M. Yoshizawa
}

\begin{abstract}
The purpose of this study is to evaluate the accessory pathway in the Wolff-Parkinson-White (WPW) syndrome. We measured magnetocardiograms (MCGs) for normal subjects and patients with the WPW syndrome using SQUID magnetometer. It is generally difficult to estimate the accessory pathway by visualization of the current-density distribution (VCDD) because the VCDD has a wide range. Independent component analysis (ICA) is a useful method for separating independent signals from overlapping signals. The source estimation of the accessory pathway was done by the VCDD using ICA. The effect of the accessory pathway was extracted by ICA, and the activity of the accessory pathway was accurately estimated. ICA was able to extract the effect of the accessory pathway. It is confirmed that ICA is a useful method to estimate the VCDD of MCG with WPW syndrome patients.
\end{abstract}

Index Terms-Accessory pathway, magnetocardiogram (MCG), visualization of the current-density distribution (VCDD), WolffParkinson-White syndrome (WPW).

\section{INTRODUCTION}

M AGNETOCARDIOGRAM (MCG) measurement is useful to estimate the heart activity, and the localization of the MCG is better than that of the electrocardiogram (ECG). Recently, the clinical application of the MCG is expected. In the Wolff-Parkinson-White (WPW) syndrome, the existence of the accessory pathway often caused paroxysmal atrial tachycardia. The activity of the accessory pathway is measured as a delta wave (the signal of delta shape before the $\mathrm{R}$ wave) of the MCG. Recent therapeutic advance in cardiology, such as interventional procedures using catheters, has been adopted for an ablation of accessory pathways [1]. On this occasion, preoperative evaluation of the accessory pathway is needed for shortening of the treatment and reducing the burden on the patient. There are some MCG studies of the WPW syndrome in source estimation [2] and modeling of the WPW syndrome [3]. It is generally difficult to separate of the accessory pathway and normal conduction system by visualization of the current-density distribution (VCDD) because the VCDD has a wide range

Manuscript received October 16, 2003. This work was supported by the Tokyo Denki University Frontier Research and Development Center.

K. Kobayashi and M. Yoshizawa are with the Faculty of Engineering, Iwate University, Iwate 020-8551, Japan (e-mail: kobaya@iwate-u.ac.jp; yosizawa@ iwate-u.ac.jp).

Y. Uchikawa is with the Faculty of Science and Engineering, Tokyo Denki University, Saitama 350-0394, Japan (e-mail: uchikawa@f.dendai.ac.jp).

K. Nakai is with Laboratory Medicine, Iwate Medical University, Iwate 020-0113, Japan (e-mail: knakai@iwate-med.ac.jp).

Digital Object Identifier 10.1109/TMAG.2004.828990

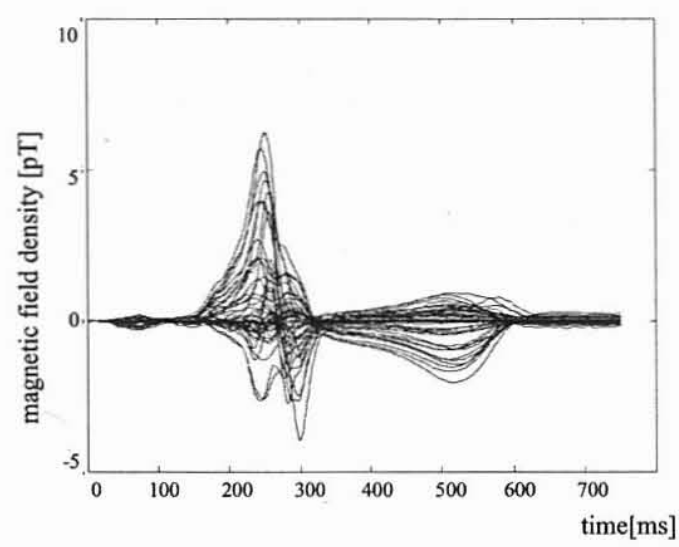

Fig. 1. MCG waveforms of the WPW syndrome patient.

TABLE I

CONTRIBUtion RATIO [\%] OF PCA With THE Two WPW SYNDROME PATIENTS AND A NORMAL SUBJECT

\begin{tabular}{c|c|c|c}
\hline & WPW1 & WPW2 & normal \\
\hline $1^{\text {s }}$ & 79.58 & 71.01 & 80.63 \\
\hline $2^{\text {nd }}$ & 17.66 & 25.28 & 13.21 \\
\hline $3^{\text {rd }}$ & 1.67 & 2.38 & 4.01 \\
\hline $4^{\text {th }}$ & 0.46 & 0.84 & 1.24 \\
\hline $5^{\text {th }}$ & 0.36 & 0.26 & 0.53 \\
\hline $6^{\text {th }}$ & 0.10 & 0.06 & 0.16 \\
\hline
\end{tabular}

[4]-[6]. Independent component analysis (ICA) is a useful method to separate independent signals from overlapping signals.

In this paper, we measured MCG of normal subjects and WPW syndrome patients. The source estimation of the accessory pathway was done by the VCDD using ICA.

\section{METHOD}

MCGs of normal subjects and WPW syndrome patients were measured by SQUID magnetometer. Standard 12-lead ECGs were also measured. The MCGs were measured in 48 positions in the 4-cm interval. A bandpass filter was used in the range of $0.1-80 \mathrm{~Hz}$. All magnetic data were averaged for about 150 times at each position. The reference signal for averaging was the $R$ wave of the ECG (lead II).

First, principal component analysis (PCA) was applied to the MCG as preprocessing. The components of under $0.1 \%$ contribution ratio were cut down in order to contract the data. This 

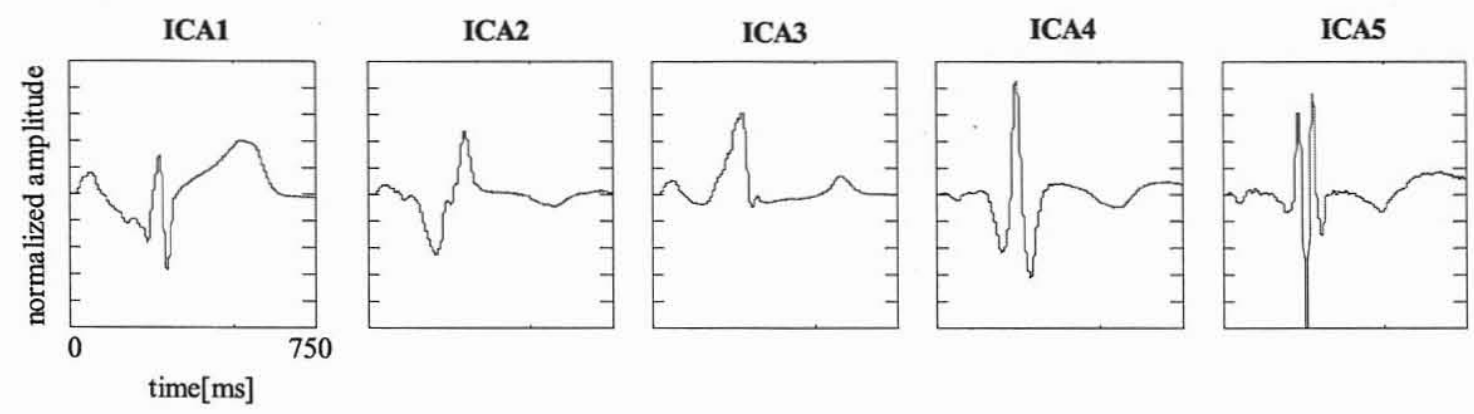

Fig. 2. MCG waveforms (ICAI-ICA5) separated by ICA of the WPW syndrome patient (WPW1 in Table I).

threshold was experientially decided from the noise level of the system. Second, ICA was performed to some dominant components. The parameters of ICA were decided the minimum method of the cross correlation [7], [8]. In order to extract the activity of the accessory pathway, the component of which the correlation was the highest for the ECG waveform of the normal subject, was removed. The waveform was reconstituted from the remaining components. Finally, the VCDD was calculated using reconstituted data.

The reason we compared the ECG waveform of the normal subject and the ICA waveform of the WPW syndrome patient is shown in the following. The comparison of the MCG waveform of the normal subject and the ICA waveform of the WPW syndrome patient is desirable, but the MCG waveform differs greatly from the measuring position. It is difficult to decide which waveform may be utilized. On the other hand, the ECG waveform (read II) of the normal subject resembles very well. Therefore, the ECG is effective as a comparative waveform.

\section{RESULTS}

Fig. 1 shows an example of the MCG waveforms recorded from the WPW syndrome patient. In these waveforms, the delta wave that is features of a WPW syndrome exists.

Table I shows the contribution ratio (\%) of PCA with the two WPW syndrome patients and a normal subject. The components of under $0.1 \%$ contribution ratio (both WPW1 and WPW2) were the sixth component or less, so these components were cut down. ICA was performed to fifth dominant components.

Fig. 2 shows the MCG waveforms (ICA1-ICA5) separated by the ICA of the WPW syndrome patient. The ICA4 waveform is similar to the waveform of the normal subject.

Fig. 3 shows the ICA4 waveform and the ECG waveform of the normal subject. The correlation coefficient from the $\mathrm{P}$ wave to the $S$ wave $(100-320 \mathrm{~ms})$ is 0.80 . In order to extract the effect of the accessory pathway, the ICA4 waveform that was similar to the waveform of the normal subject was removed, and the reconstruction with the MCG waveform (ICA1235) was done using ICA1, ICA2, ICA3, and ICA5.

Fig. 4 shows the reconstituted MCG waveforms (ICA1235) of the WPW syndrome patient. We can see that the effect of the accessory pathway is being extracted by ICA.

Fig. 5 shows the VCDD of the MCG with the WPW syndrome patient (WPW1), (a) without ICA and (b) with ICA. Each figure shows the current density of about 8 -cm under body surface, and

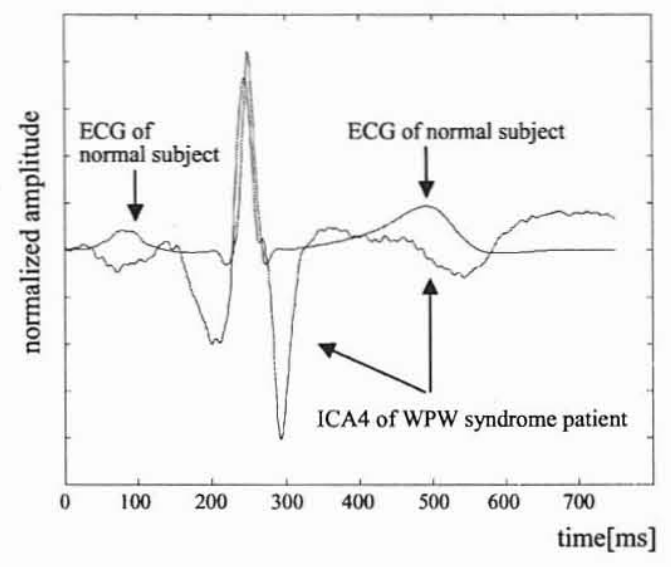

Fig. 3. ECG waveform of the normal subject and the ICA4 waveform of the WPW syndrome patient.

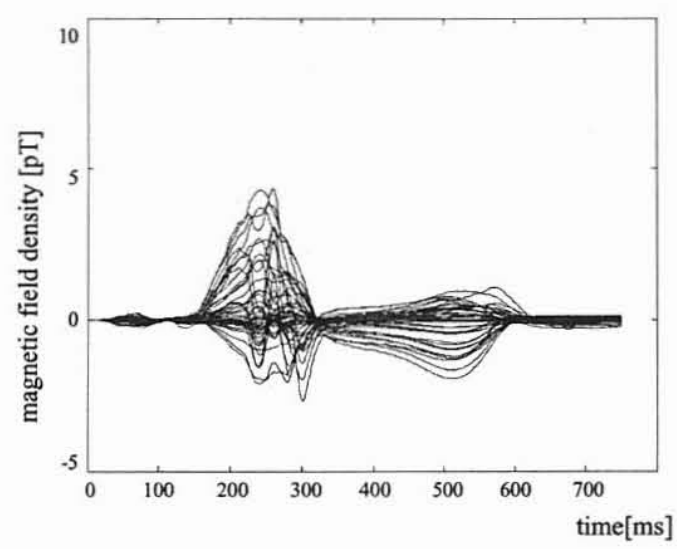

Fig. 4. Reconstituted MCG waveforms (ICA1235) of the WPW syndrome patient with ICA.

the current density of this plane is the highest. The VCDDs show the time in which the delta wave exists. The $x$ axis and $y$ axis show the measuring point. The strength of the current density is shown in the intensity. The white part has shown the place with the high strength. The triangular mark has shown the direction of the current density.

In both Fig. 5(a) and (b) at $160 \mathrm{~ms}$, the current density exists for the center of D2, D3, E2, and E4. In Fig. 5(a) at $180 \mathrm{~ms}$, the current density exists for the wide range between D2 and E6. The position of the maximum current density is between D4 


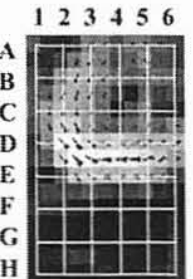

160

123456

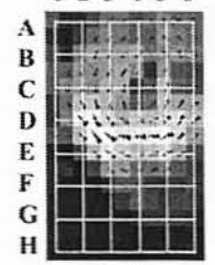

160

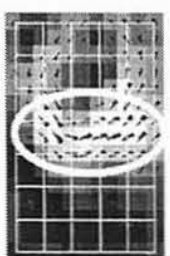

180

(a)

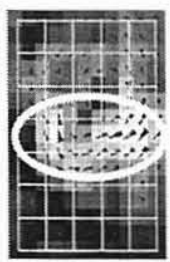

180

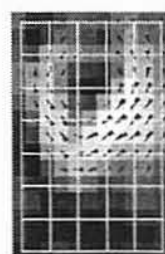

200

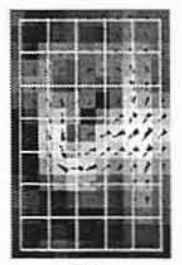

200

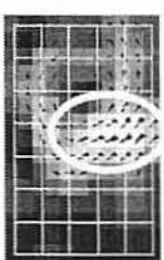

220

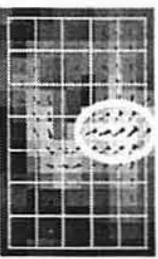

220 (b)

Fig. 5. VCDD of MCG with the WPW syndrome patient. (a) VCDD without ICA. (b) VCDD with ICA.

and D5. On the other hand, in Fig. 5(b) at $180 \mathrm{~ms}$, the current density has been localized at the center of D2, D3, E2, and E4, and between D5 and E6.

In Fig. 5(a) at $220 \mathrm{~ms}$, the current density exists for the wide range between D4 and E6, and the position of the current density is moved from the position at $180 \mathrm{~ms}$. In Fig. 5(b) at $220 \mathrm{~ms}$, the current density has been localized between D5 and E6, and the position of the current density is the same position at $180 \mathrm{~ms}$.

The effect of the accessory pathway is extracted by ICA and the VCDD is localized.

\section{Discussion}

The activity of the accessory pathway of a WPW syndrome patient has been localized in the time domain of the delta wave [9], [10]. In comparison with the original signal, the VCDD was localized by ICA. Then, the current density existed for the same position for longer time. This result indicates that the effect of the accessory pathway was extracted by ICA, and the activity of the accessory pathway was more accurately estimated. This method is expected for clinical applications.

\section{CONCLUSION}

We measured the MCG of normal subjects and WPW syndrome patients. In order to analyze the accessory pathway of a WPW syndrome patient, the VCDD by ICA was done. ICA was able to extract the effect of the accessory pathway. The activity of the accessory pathway was more accurately estimated. These findings demonstrated that the VCDD by ICA were valuable for the analysis of the heart activity.

\section{REFERENCES}

[1] K. Grauer, A Practical Guide to ECG Interpretation. Tokyo, Japan: Medical View, 1995.

[2] J. Nenonen, T. Katila, M. Leinio, J. Montone, M. Makijarvi, and P. Siltanen, "Magnotocardiographic functional localization using current multipole models," IEEE Trans. Biomed. Eng., vol. 38, pp. 648-657. Apr. 1991.

[3] Y. Uchikawa and S. N. Erne, "Modeling the Wolfe-Parkinson-White syndrome for magnetocardiography," in Proc. Biomagnetism, 1987, pp. 322-325.

[4] K. Nakai, M. Yoshizawa, K. Kobayashi, Y. Nakamura, A. Suwabe, K. Kawazoe, and Y. Uchikawa, "Newly developed synthetic magnetometry on magnetocardiography can visualize three dimensional infracted and ischemic myocardium by current density map," in Proc. 13th Int. Conf. Biomagnetism, 2002, pp. 584-586.

[5] J. Vrba, "Magnetoencephalography: The art of finding a needle in a haystack," Phys. C: Superconduct., vol. 368, pp. 1-9, 2002.

[6] K. Sekihara and B. Scholz, "Generalized wiener estimation of three-dimensional current distribution from biomagnetic measurements," IEEE Trans. Biomed. Eng., vol. 43, pp. 281-291, Feb. 1996.

[7] J. F. Cardoso and A. Souloumiac, "Jacobi angles for simultaneous diagonalization," SIAM J. Mater. Anal. Appl., vol. 17, no. 1, p. 161, 1996.

[8] A. Hyvarinen, J. Karhunen, and E. Oja, Independent Component Analysis. New York: Wiley, 2001.

[9] Y. Gojima and K. Ohbayashi, Electrocardiogram ABC. Tokyo, Japan: Japan Medical Association, 1992.

[10] M. Obata and K. Sugimoto, Internal Medicine. Tokyo, Japan: Asakura, 1977. 